Tôhoku Math. Journ. $28(1976)$, 553-561.

\title{
A DISTORTION THEOREM IN $K$-QUASICONFORMAL MAPPINGS OF THE $n$-BALL
}

\author{
KAZUO IKомA
}

(Received June 3, 1975)

\begin{abstract}
As is well known, the plane Teichmüller's modulus theorem which estimates for the modulus of the plane ring separating a pair of points 0 and $\gamma$ from $\delta$ and $\infty$ played an important role in dealing with distortion problems in function theory. Lehto-Virtanen [4] and A. Mori [5] modified this theorem so as to be suitable for further applications to distortion problems, that is to say, they estimated for the modulus of the plane ring separating a pair of points $\alpha$ and $\beta$ from 0 and $\infty$. The main point of the proof for this modification is that a ring separating $\alpha$ and $\beta$ from 0 and $\infty$ is doubled by means of two branches of the inverse of the mapping $z=w^{2}$ and then a ring whose boundary components are separated by doubled rings is transformed into another ring to which the plane Teichmüller's modulus theorem can be applied.

Now, in order to estimate for the modulus of a ring in $n$-space $(n \geqq 3$ ) separating a pair of points $\alpha$ and $\beta$ from 0 and $\infty$, the ring is to be transformed into another ring so that one may be able to use Teichmüller's modulus theorem in $n$-space which estimates for the modulus of a ring separating a pair of points 0 and $\gamma$ from $\delta$ and $\infty$. To this purpose, an appropriate auxiliary $K$-quasiconformal mapping is required, where $K$ can not be equal to 1 . The reason can be seen from the above mentioned situation in the 2-dimensional case and the familiar result*) that every 1-quasiconformal mapping of a domain in space is nothing but a restriction of a Möbius transformation to the domain. In this paper, we estimate in Theorem 1, for the modulus of a ring in $n$ space separating a pair of points $\alpha$ and $\beta$ from 0 and $\infty$, and as its application, show in Theorem 2, a distortion result of the Hölder type for $K$-quasiconformal mappings of the unit ball in $n$-space.
\end{abstract}

1. The modulus of a ring and $K$-quasiconformality. A domain $R$ in the Möbius $n$-space**) is called a ring if its complement has exactly

*) See, for instance, Rešetnjak [7].

**) The Möbius $n$-space means the one point compactification of the euclidean $n$-space obtained by adding the point at infinity. 
two components, which we denote by $C_{0}$ and $C_{1}$. Then, the boundary of $R$ has also two components, i.e. $B_{0}=C_{0} \cap \bar{R}$ and $B_{1}=C_{1} \cap \bar{R}$, where $\bar{R}$ is the closure of $R$.

The modulus of a ring $R$ has been defined in the various but equivalent ways. Here, we shall use the definition in terms of the extremal length, and denote by $m_{k}$ the $k$-dimensional Lebesgue measure. For each real-valued and non-negative Borel measurable function $\rho(x)$ of $R$, let

$$
L(\rho)=\inf _{\gamma} \int_{r} \rho(x) d s \quad\left(d s=d m_{1}\right),
$$

where the infimum is taken over all locally rectifiable curves $\gamma$ joining $B_{0}$ and $B_{1}$ in $R$. And put

$$
S(\rho)=\inf _{\Sigma} \int_{\Sigma} \rho(x)^{n-1} d \sigma \quad\left(d \sigma=d m_{n-1}\right),
$$

where the infimum is taken over all compact piecewise smooth $(n-1)$ dimensional hyper-surfaces $\Sigma$ separating $B_{0}$ and $B_{1}$ in $R$. Further we put

$$
V(\rho)=\int_{R} \rho(x)^{n} d \omega \quad\left(d \omega=d m_{n}\right) .
$$

Then it has been shown by Krivov [3] that

$$
\sup _{\rho} \frac{L(\rho)^{n}}{V(\rho)}=\inf _{\rho} \frac{V(\rho)^{n-1}}{S(\rho)^{n}} \text {. }
$$

The quantities

$$
\left(\sigma_{n-1} \sup _{\rho} \frac{L(\rho)^{n}}{V(\rho)}\right)^{1 /(n-1)} \text { and }\left(\sigma_{n-1} \inf _{\rho} \frac{V(\rho)^{n-1}}{S(\rho)^{n}}\right)^{1 /(n-1)},
$$

which are equal to each other, are called the modulus of $R$ and are denoted by $\bmod R$, where $d \sigma_{n-1}$ denotes the $(n-1)$-measure of the surface of the unit $n$-ball.

The following $n$-dimensional versions of properties of moduli have been shown by Mostow [6], which will be used later on.

The superadditivity of the modulus of a ring. Let $R$ be a ring and let $R_{1}, R_{2}, \cdots, R_{m}$ be mutually disjoint rings each separating two boundary components of $R$, then

$$
\bmod R_{1}+\bmod R_{2}+\cdots+\bmod R_{m} \leqq \bmod R,
$$

which reduces to the monotonicity when $m=1$.

A ring whose complementary components are the unit ball $\{x|| x \mid \leqq 1\}$ 
and the ray $\left\{x \mid c \leqq x_{1} \leqq \infty, x_{2}=x_{3}=\cdots=x_{n}=0\right.$, where $\left.1<c\right\}$ is called the Grötzsch ring in space and is denoted by $R_{G}(c)$. This is equivalent 1-quasiconformally to the ring whose complement consists of the segment $\left\{x \mid 0 \leqq x_{1}=1 / c, x_{2}=\cdots=x_{n}=0\right\}$ and the exterior $\{x|| x \mid \geqq 1\}$ of the unit ball.

Grötzsch's modulus theorem. Let $R$ be a ring whose complementary components are $C_{0}$ and $C_{1}$. If $C_{0}$ contains the ball $\{x|| x \mid \leqq a\}$ and $C_{1}$ contains some point at distance $b$ from the origin, then

$$
\bmod R \leqq \log \Phi_{n}\left(\frac{b}{a}\right),
$$

where $\log \Phi_{n}(c)$ means the modulus of the Grötzsch ring $R_{G}(c)$.

Teichmuiller's modulus theorem. If $C_{0}$ contains the origin and a point $\alpha$ at distance $\alpha$ from the origin, and $C_{1}$ contains a point $\beta$ at distance $b$ from the origin, then

$$
\bmod R \leqq 2 \log \Phi_{n}\left(\sqrt{\frac{a+b}{a}}\right) .
$$

Next, let $D$ and $D^{\prime}$ be domains in the Möbius $n$-space. If a homeomorphism $f: D \rightarrow D^{\prime}$ satisfies the double inequality

$$
\frac{1}{K} \bmod R \leqq \bmod f(R) \leqq K \bmod R
$$

for every ring $R$ whose closure is contained in $D$, then $f$ is said to be $K$-quasiconformal.*)

Particularly, let $f: D \rightarrow D^{\prime}$ be a diffeomorphism. Let $e_{1}, e_{2}, \cdots, e_{n}$ be the unit vector in $n$-space, and be $h=l_{1} e_{1}+l_{2} e_{2}+\cdots+l_{n} e_{n}$, where $l_{1}^{2}+l_{2}^{2}+\cdots+l_{n}^{2}=|h|^{2}=1$. Put

$$
\max _{|h|=1}\left|f^{\prime}(x) h\right|=L\left(f^{\prime}(x)\right) \text { and } \min _{|h|=1}\left|f^{\prime}(x) h\right|=l\left(f^{\prime}(x)\right),
$$

where $f^{\prime}(x)$ means the derivative of $f(x)$. We here refer to a criterion (Väisälä [8], Corollary 15.4) for the $K$-quasiconformality.

LEMMA 1. A diffeomorphism $f: D \rightarrow D^{\prime}$ is $K$-quasiconformal if and only if the double inequality

$$
\frac{1}{K^{n-1}} L\left(f^{\prime}(x)\right)^{n} \leqq|J(x, f)| \leqq K^{n-1} l\left(f^{\prime}(x)\right)^{n}
$$

*) Väisälä [8] defined the modulus of a ring by the $(n-1)$-power of the quantity in the definition of the modulus mentioned above. Hence, we remark that $f$ is $K$-quasiconformal in Väisälä's sense if and only if $f$ is $K^{1 /(n-1)}$-quasiconformal by the above definition. 
holds for every $x \in D$.

2. A modification of Teichm ïller's modulus theorem. In estimating for the modulus of the plane ring separating $\alpha$ and $\beta$ from 0 and $\infty$, the ring was transformed by two branches of the inverse of the mapping $z=w^{2}$ and by an appropriate Möbius transformation into another ring to which the plane Teichmüller's modulus theorem can be applied. In order to obtain an estimation for the modulus of a ring $R$ separating $\alpha$ and $\beta$ from 0 and $\infty$ in $n$-space, we first consider the mapping

$$
x_{1}=y_{1}^{2}-y_{2}^{2}, \quad x_{2}=2 y_{1} y_{2}, \quad x_{j}=y_{j} \quad(3 \leqq j \leqq n)
$$

in the $n$-dimensional case $(n \geqq 3)$ corresponding to the mapping $z=w^{2}$ in the plane. This is clearly a diffeomorphism in a finite domain $D$ not containing the origin. Denote by $x=f(y)$ this mapping, then it is seen from some elementary computations that

$$
|J(y, f)|=4\left(y_{1}^{2}+y_{2}^{2}\right),
$$

and

$$
\frac{L\left(f^{\prime}(y)\right)^{n}}{|J(y, f)|}=\left\{\begin{array}{lll}
\left\{4\left(y_{1}^{2}+y_{2}^{2}\right)\right\}^{n / 2-1} & \text { for } & 4\left(y_{1}^{2}+y_{2}^{2}\right)>1 \\
\left\{4\left(y_{1}^{2}+y_{2}^{2}\right)\right\}^{-1} & \text { for } & 4\left(y_{1}^{2}+y_{2}^{2}\right)<1
\end{array}\right.
$$

and

$$
\frac{|J(y, f)|}{l\left(f^{\prime}(y)\right)^{n}}=\left\{\begin{array}{lll}
4\left(y_{1}^{2}+y_{2}^{2}\right) & \text { for } & 4\left(y_{1}^{2}+y_{2}^{2}\right)>1, \\
\left\{4\left(y_{1}^{2}+y_{2}^{2}\right)\right\}^{1-n / 2} & \text { for } & 4\left(y_{1}^{2}+y_{2}^{2}\right)<1 .
\end{array}\right.
$$

Hence we have

$$
\sup _{y \in D} \frac{L\left(f^{\prime}(y)\right)^{n}}{|J(y, f)|}=\infty \quad \text { and } \sup _{y \in D} \frac{|J(y, f)|}{l\left(f^{\prime}(y)\right)^{n}}=\infty,
$$

from which Lemma 1 implies that the mapping $x=f(y)$ in (2.0) is not quasiconformal in $D$. Hence follows that there is neither finite upper bound nor positive lower bound for $\left(\bmod R^{\prime} / \bmod R\right)$ when a $\operatorname{ring} R$ varies. Consequently, we can not use this mapping in our estimate.

Then, we give our eyes to the mapping

$$
x_{1}=\frac{y_{1}^{2}-y_{2}^{2}}{\sqrt{y_{1}^{2}+y_{2}^{2}}}, \quad x_{2}=\frac{2 y_{1} y_{2}}{\sqrt{y_{1}^{2}+y_{2}^{2}}}, \quad x_{j}=y_{j} \quad(3 \leqq j \leqq n) .
$$

Because, its inverse mapping consists of two branches $y=y_{+}(x)$ and $y=y_{-}(x)$ :

$$
y_{1}=r \cos \frac{\theta}{2}, \quad y_{2}=r \sin \frac{\theta}{2}, \quad y_{j}=x_{j} \quad(3 \leqq j \leqq n)
$$


for $-\pi \leqq \theta<\pi$ and for $\pi \leqq \theta<3 \pi$, respectively, and these branches are 2-quasiconformal as will be proved below and further a ring considered is doubled by means of these branches, where $\left(r, \theta, x_{3}, \cdots, x_{n}\right)$ is the cylindrical coordinates of a point $x$ of the euclidean $n$-space. Each of homeomorphisms $y=y_{+}(x):\{x \mid-\pi<\theta<\pi\} \rightarrow\{y \mid-\pi / 2<\varphi<$ $\pi / 2\}$ and $y=y_{-}(x):\{x \mid \pi<\theta<3 \pi\} \rightarrow\{y \mid \pi / 2<\varphi<3 \pi / 2\}$ is diffeomorphism and is called a folding. After some elementary calculations, we have for $y(x)$ of (2.1),

$$
|J(x, y)|=\frac{1}{2} \quad \text { and } \quad\left|y^{\prime}(x) h\right|^{2}=1-\frac{3}{4}\left(l_{1} \sin \theta-l_{2} \cos \theta\right)^{2},
$$

so that

$$
L\left(y^{\prime}(x)\right)=1 \quad \text { and } \quad l\left(y^{\prime}(x)\right)=\frac{1}{2}
$$

Therefore we have

$$
\frac{|J(x, y)|}{l\left(y^{\prime}(x)\right)^{n}}=2^{n-1} \text { and } \frac{L\left(y^{\prime}(x)\right)^{n}}{|J(x, y)|}=2 \leqq 2^{n-1},
$$

from which Lemma 1 yields that both branches $y=y_{+}(x)$ and $y=y_{-}(x)$ are 2-quasiconformal.

Now, we prove, by the aid of these branches, the following theorem which means a modification of Teichmüller's modulus theorem stated in Section 1.

THEOREM 1. If a ring $R$ in $n$-space separates a pair of points $\alpha$ and $\beta$ from the origin and the point at infinity, then

$$
\bmod R \leqq 2 \log \Phi_{n}\left(\frac{\sqrt{2\left(|\alpha|^{2}+|\beta|^{2}\right)}}{|\alpha-\beta|}\right) .
$$

Proof. We first choose the coordinate (hyper) plane $x_{2}=0$ so as to pass through $\alpha$ and $\beta$, and take the $x_{1}$-axis such that $\alpha$ lies on the positive axis.

Now, denote by $R^{\prime}$ the image of $R$ obtained by the branch $y=y_{+}(x)$, and by $R^{\prime \prime}$ the image of $R$ by $y=y_{-}(x)$ in (2.1). Let $C_{0}^{\prime}$ and $C_{0}^{\prime \prime}$ be the bounded components of the complement of $R^{\prime}$ and $R^{\prime \prime}$, respectively, then the complement of $C_{0}^{\prime} \cup C_{0}^{\prime \prime}$ forms a ring, which is denoted by $R_{0}$. Since both $R^{\prime}$ and $R^{\prime \prime}$ separate one of $C_{0}^{\prime}$ and $C_{0}^{\prime \prime}$ from the other, the superadditivity of the modulus of a ring implies that

$$
\bmod R^{\prime}+\bmod R^{\prime \prime} \leqq \bmod R_{0} \text {. }
$$

Using 2-quasiconformality of branches in (2.1), we have 


$$
\bmod R^{\prime}=\bmod R^{\prime \prime} \geqq \frac{1}{2} \bmod R,
$$

so that

$$
\bmod R \leqq \bmod R_{0} \text {. }
$$
mation

Next, transform $R_{0}$ into another ring $R_{0}^{\prime}$ by the Möbius transfor-

$$
\left\{\begin{array}{l}
w_{1}=\frac{\sum_{k=1}^{n} y_{k}^{2}-a_{1}^{2}}{\left(y_{1}+a_{1}\right)^{2}+\sum_{k=2}^{n} y_{k}^{2}} \\
w_{j}=\frac{4 a_{1} y_{j}}{\left(y_{1}+a_{1}\right)^{2}+\sum_{k=2}^{n} y_{k}^{2}} \quad(2 \leqq j \leqq n),
\end{array}\right.
$$

where $\alpha=\left(\alpha_{1}, 0, \cdots, 0\right)$. Then

$$
\bmod R_{0}=\bmod R_{0}^{\prime} \text {. }
$$

Denote by $\alpha_{+}^{\prime}, \beta_{+}^{\prime}$ the image points of $\alpha=\left(a_{1}, 0, \cdots, 0\right), \beta=\left(b_{1}, 0\right.$, $\left.b_{3}, \cdots, b_{n}\right)$ by the branch $y=y_{+}(x)$ in (2.1), then we have $\alpha_{+}^{\prime}=\alpha$ and $\beta_{+}^{\prime}=\beta$. And denote by $\alpha_{-}^{\prime}, \beta_{-}^{\prime}$ the image points of $\alpha, \beta$ by the branch $y=y_{-}(x)$ in (2.1), then we get $\alpha_{-}^{\prime}=\left(-a_{1}, 0, \cdots, 0\right)$ and $\beta_{-}^{\prime}=\left(-b_{1}, 0\right.$, $\left.b_{3}, \cdots, b_{n}\right)$. Further, let $\alpha_{+}^{\prime \prime}, \beta_{+}^{\prime \prime}$ be the image points of $\alpha_{+}^{\prime}, \beta_{+}^{\prime}$ by the mapping (2.3), then we have

$$
\begin{aligned}
& \alpha_{+}^{\prime \prime}=(0, \cdots, 0)=\text { the origin and } \\
& \beta_{+}^{\prime \prime}=\left(\frac{b_{1}^{2}+\sum_{k=3}^{n} b_{k}^{2}-a_{1}^{2}}{\left(b_{1}+a_{1}\right)^{2}+\sum_{k=3}^{n} b_{k}^{2}}, 0, \frac{4 a_{1} b_{j}}{\left(b_{1}+a_{1}\right)^{2}+\sum_{j=3}^{n} b_{k}^{2}}\right), \quad 3 \leqq j \leqq n .
\end{aligned}
$$

And let $\alpha_{-}^{\prime \prime}, \beta_{-}^{\prime \prime}$ be the image points of $\alpha_{-}^{\prime}, \beta_{-}^{\prime}$ by the mapping (2.3), then we get

$$
\begin{aligned}
& \alpha_{-}^{\prime \prime}=\text { the point at infinity and } \\
& \beta_{-}^{\prime \prime}=\left(\frac{b_{1}^{2}+\sum_{k=3}^{n} b_{k}^{2}-a_{1}^{2}}{\left(-b_{1}+a_{1}\right)^{2}+\sum_{k=3}^{n} b_{k}^{2}}, 0, \frac{4 a_{1} b_{j}}{\left(-b_{1}+a_{1}\right)^{2}+\sum_{k=3}^{n} b_{k}^{2}}\right), \quad 3 \leqq j \leqq n .
\end{aligned}
$$

Since $R_{0}^{\prime}$ separates the points the origin and $\beta_{+}^{\prime \prime}$ from the point at infinity and $\beta_{-}^{\prime \prime}$, the $n$-dimensional version of Teichmüller's modulus theorem stated in Section 1 yields that 


$$
\begin{aligned}
\bmod R_{0}^{\prime} & \leqq 2 \log \Phi_{n}\left(\sqrt{\frac{\left|\beta_{+}^{\prime \prime}\right|+\left|\beta_{-}^{\prime \prime}\right|}{\left|\beta_{+}^{\prime \prime}\right|}}\right) \\
& =2 \log \Phi_{n}\left(\sqrt{\frac{2\left(a_{1}^{2}+b_{1}^{2}+\sum_{k=3}^{n} b_{k}^{2}\right)}{\left(-b_{1}+a_{1}\right)^{2}+\sum_{k=3}^{n} b_{k}^{2}}}\right) \\
& =2 \log \Phi_{n}\left(\sqrt{\frac{2\left(|\alpha|^{2}+|\beta|^{2}\right)}{|\alpha-\beta|}}\right) .
\end{aligned}
$$

Combining it with (2.2) and (2.4), we have the desired estimation.

3. A distortion result in $K$-quasiconformal mappings of the ball.

In applying Theorem 1 to $K$-quasiconformal mappings in $n$-space, an estimate for $\Phi_{n}(a) / a$ is required. $\Phi_{2}(a) / a \leqq 4$ is well known, and $\Phi_{3}(a) / a \leqq$ $4 \sqrt{2} e^{\pi / 4}$ was shown by Gehring [1]. An $n$-dimensional version of such an estimation was obtained by us as follows.

LEMMA 2. (Ikoma [2]). $\Phi_{n}(a) \leqq \lambda_{n} a$, where

$$
\lambda_{n}=4\left[\exp \left\{\frac{\pi}{4} \cot \frac{\pi}{2(n-1)}\right\}\right] \prod_{k=1}^{n-2}\left\{\sin \frac{k \pi}{2(n-1)}\right\}^{(-1)^{k}-\cos (k \pi /(n-1))} .
$$

Then the following distortion theorem is proved by applying Grötzsch's modulus theorem and Theorem 1 and Lemma 2.

THEOREM 2. Let $y=y(x)$ be any K-quasiconformal mapping of the unit ball in $n$-space onto itself normalized $y(0)=0$. Then, for every pair of points $\alpha, \beta$ with $|\alpha| \leqq 1,|\beta| \leqq 1$,

$$
|y(\alpha)-y(\beta)| \leqq 2 \lambda_{n}|\alpha-\beta|^{1 / 2 K} \text {. }
$$

Proof. Since $|y(\alpha)-y(\beta)| \leqq|y(\alpha)|+|y(\beta)| \leqq 2$, there holds for $|\alpha-\beta| \geqq 1 / \lambda_{n}$,

$$
|y(\alpha)-y(\beta)| \leqq 2<2 \lambda_{n}\left(\frac{1}{\lambda_{n}}\right)^{1 / K}<2 \lambda_{n}|\alpha-\beta|^{1 / 2 K}
$$

Hence, our theorem has been proved for $|\alpha-\beta| \geqq 1 / \lambda_{n}$. Since it is trivial for $|\alpha-\beta|=0$, it suffices to prove the theorem for the case $0<|\alpha-\beta|<1 / \lambda_{n}$.

First, we prove the theorem for the case $|\alpha+\beta| \leqq 1$. Since the spherical ring $A=\{x|| \alpha-\beta|/ 2<| x-(\alpha+\beta) / 2 \mid<1 / 2\}$ is contained in the unit ball $|x|<1$, the image $y(A)$ of $A$ is also contained in $|y|<1$. Therefore, $y(A)$ is contained in the ball $|y-y(\alpha)|<2$, so that the one component of the complement of $y(A)$ contains both $y(\alpha)$ and $y(\beta)$, and the other component of that contains $\{y|| y-y(\alpha) \mid \geqq 2\}$. Consequently, 
we have by Grötzsch's modulus theorem,

$$
\bmod y(A) \leqq \log \Phi_{n}\left(\frac{2}{|y(\alpha)-y(\beta)|}\right) .
$$

Taking the modulus condition

$$
\frac{1}{K} \bmod A=\frac{1}{K} \log \frac{1}{|\alpha-\beta|} \leqq \bmod y(A)
$$

into account, we have

$$
\log \frac{1}{|\alpha-\beta|^{1 / K}} \leqq \log \Phi_{n}\left(\frac{2}{|y(\alpha)-y(\beta)|}\right) .
$$

Using the above Lemma 2, we get

$$
\frac{1}{|\alpha-\beta|^{1 / K}}<\frac{2 \lambda_{n}}{|y(\alpha)-y(\beta)|},
$$

so that

$$
|y(\alpha)-y(\beta)|<2 \lambda_{n}|\alpha-\beta|^{1 / K}<2 \lambda_{n}|\alpha-\beta|^{1 / 2 K} .
$$

Finally, we consider the case $|\alpha+\beta|>1$. The ring $A$ in this case extends over the exterior to the ball $|x| \leqq 1$, and so we extend $y=y(x)$ to $y^{*}(x)$ of $|x|<\infty$ to $|y|<\infty K$-quasiconformally. That is to say, extend $y(x)$ to $|x|>1$ by

$$
y^{*}(x)=\left\{\begin{array}{l}
y(x), \quad|x| \leqq 1, \\
y\left(\frac{x}{|x|^{2}}\right) /\left|y\left(\frac{x}{|x|^{2}}\right)\right|^{2}, \quad|x|>1,
\end{array}\right.
$$

then $y^{*}(x)$ becomes a $K$-quasiconformal mapping of $|x|<\infty$ to $|y|<\infty$.

Now, since the image $\operatorname{ring} y^{*}(A)$ of $A$ separates the origin and $\infty$ from $y(\alpha)$ and $y(\beta)$, we obtain by Theorem 1 ,

$$
\bmod y^{*}(A) \leqq 2 \log \Phi_{n}\left(\frac{\sqrt{2\left(|y(\alpha)|^{2}+|y(\beta)|^{2}\right)}}{|y(\alpha)-y(\beta)|}\right) .
$$

Using the preceding Lemma 2 again, we have

$$
\begin{aligned}
\bmod y^{*}(A) & \leqq 2 \log \Phi_{n}\left(\frac{\sqrt{2} \cdot \sqrt{2}}{|y(\alpha)-y(\beta)|}\right) \\
& <2 \log \left(\frac{2 \lambda_{n}}{|y(\alpha)-y(\beta)|}\right)
\end{aligned}
$$

Together this with the modulus condition in the definition of $K$-quasiconformality, we get 


$$
\log \frac{1}{|\alpha-\beta|^{1 / K}}<2 \log \left(\frac{2 \lambda_{n}}{|y(\alpha)-y(\beta)|}\right),
$$

whence the required result follows.

\section{REFERENCES}

[1] F. W. GeHring, Symmetrization of rings in space, Trans. Amer. Math. Soc., 101 (1961), 499-519.

[2] K. Iкома, An estimate for the modulus of the Grötzsch ring in $n$-space, Bull. Yamagata Univ. (Nat. Sci.) 6 (1967), 395-400.

[3] V. V. KRIvov, Some properties of moduli in space, Soviet Math. Dokl., 5 (1964), 113117.

[4] O. Lehto and K. I. Virtanen, Quasikonforme Abbildungen, Springer, 1965, 269 pp.

[5] A. MoRI, On an absolute constant in the theory of quasiconformal mappings, J. Math. Soc. Japan, 8 (1956), 156-166.

[6] G. D. Mostow, Quasi-conformal mappings in $n$-space and the rigidity of hyperbolic space forms, Inst. Hautes Etudes Sci. Publ. Math. 34 (1968), 53-104.

[7] JU. G. REŠETNJAK, On the stability of conformal mappings in multidimensional spaces (Russian), Sibirsk. Mat. Ž. 8 (1967), 91-114.

[8] J. VäISÄL̈̈, Lectures on $n$-dimensional quasiconformal mappings, Lecture Notes in Math. 229, Springer, 1971, 144 pp.

DePaRTMENT OF MATHEMATICS

YAMAGATA UNIVERSITY

YAMAGATA, JAPAN 
